\title{
Distributed Computing in the Modern Enterprise
}

W elcome to the Alphabet Soup edition of Queue. You are about to wade into a sea of buzzwords and acronyms, but the future of enterprise computing is on the other shore.

The articles in this issue form a coherent yet occasionally contradictory set. Each presents a view of key technical solutions to the computing problems of the modern enterprise. Together, they describe a very important part of the future of computing for and by the enterprise. The authors emphasize different aspects. There remain disagreements about details and even about fundamental approaches. We may have several men describing an elephant, or there might actually be multiple elephants.

Firms are striving to increase the flexibility of developing applications using standards to achieve interoperability, and to manage their infrastructure resources (processors, networks, storage, applications) efficiently by taking advantage of new business models and system management techniques.

Most of the new approaches are service-based. They include grid, Web services, service-oriented architectures, utility computing, and software as a service.

Enterprises are adopting new approaches to distributed computing to meet old and new needs. The traditional problems include legacy integration (keeping the old stuff running either because it works or because no one knows how it works) and adapting to changes in platforms and computing environments. New problems arise from mergers, business restructuring, and outsourcing (which require frequent disintegration and reintegration of systems); increased concerns for security and business continuity; and new possibilities (such as services offered by new vendors). Enterprises must also handle stressful requirements, such as supporting peak loads for technical computing and running massive farms of web servers.

Why is this happening now? Companies recognize that clean IT implementations are essential for long-term viability of the solution and the firm. Hacking got us into many of the troubles we face today. Luckily, computing resources to support general-purpose solutions (XML encodings and many layers of standards-based software) are now affordable.

\author{
Grids, SOAs, \\ WEB SERVICES, \\ AND BEYOND
}

Ian Foster (one of the originators of grid and co-author of the seminal book on the subject) and Steven Tuecke (another

grid pioneer) present a view of the distributed computing landscape (page 26), driven by a vision of the shift from traditional vertical integration (all functions on a single computer or under single control) to horizontal integration (functions may be distributed among different machines or domains for efficiency or necessity).

Dean Jacobs presents his take on a very hot trend in the industry right now, software as a service (SaS) (page 36). The customer cares only about the interfaces and the service levels, so the provider can control the development approach and the deployment environment for optimal results and an on-demand solution. Venture capitalists attest to the flood of ideas and funded companies in this space, and CIOs of large companies have recently said they expect to shift significant percentages of their software spending from traditional licenses to SaS.

Pankaj Kumar discusses a new generation of standards for IT management (page 44). Managing complicated, federated, distributed systems is inherently difficult. The story is complex and the acronyms get deep. Although it is too early to tell which standards will dominate practice, some will almost certainly have great impact on how enterprises manage their information technology.

Paul Strong discusses the advantages of running a grid within a firm with a set of new technologies running in a controlled environment (page 50). His article describes how to manage a complex and changing environment through virtualization, abstraction, and automation.

Enjoy the issue-you may soon be pursuing one or more of the paths described here. $Q$

STUART FELDMAN is vice president of On Demand Business Transformation research for IBM. He was a member of the original Unix research team and is best known as the creator of the Make configuration management system, as well as the author of the first Fortran-77 compiler. He earned his Ph.D. in applied mathematics from M.I.T. 